Internet Engineering Task Force (IETF)

Request for Comments: 7805

Obsoletes: 675721761813816879896

10786013

Updates: 7414

Category: Informational

ISSN : 2070-1721
A. Zimmermann

W. Eddy

MTI Systems

L. Eggert

NetApp

April 2016

\title{
Moving Outdated TCP Extensions and TCP-Related Documents to Historic or Informational status
}

Abstract

This document reclassifies several TCP extensions and TCP-related documents that either have been superseded, have never seen widespread use, or are no longer recommended for use to "Historic" status. The affected documents are RFCs 675, 721, 761, 813, 816, 879, 896, 1078, and 6013. Additionally, this document reclassifies RFCs 700, 794, 814, 817, 872, 889, 964, and 1071 to "Informational" status.

Status of This Memo

This document is not an Internet Standards Track specification; it is published for informational purposes.

This document is a product of the Internet Engineering Task Force (IETF). It represents the consensus of the IETF community. It has received public review and has been approved for publication by the Internet Engineering Steering Group (IESG). Not all documents approved by the IESG are a candidate for any level of Internet Standard; see section 2 of RFC 5741.

Information about the current status of this document, any errata, and how to provide feedback on it may be obtained at http://www.rfc-editor.org/info/rfc7805. 
Copyright Notice

Copyright (c) 2016 IETF Trust and the persons identified as the document authors. All rights reserved.

This document is subject to BCP 78 and the IETF Trust's Legal Provisions Relating to IETF Documents

(http://trustee.ietf.org/license-info) in effect on the date of publication of this document. Please review these documents carefully, as they describe your rights and restrictions with respect to this document. Code Components extracted from this document must include Simplified BSD License text as described in section $4 . e$ of the Trust Legal Provisions and are provided without warranty as described in the Simplified BSD License.

Table of Contents

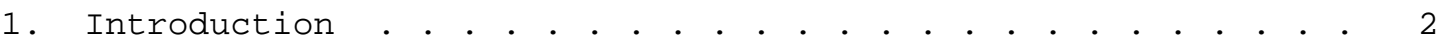

2. Status Changes . . . . . . . . . . . . . . . . . . . . . . . 3

2.1. Moving to "Historic" Status . . . . . . . . . . . . . . . 3

2.2. Moving to "Informational" Status • • • • • • • • . • . . 4

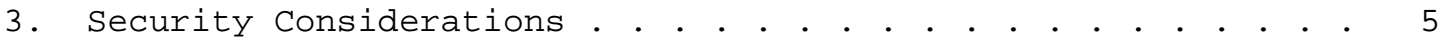

4. References . . . . . . . . . . . . . . . . . . . . . . . . 5

4.1. Normative References . . . . . . . . . . . . . . . . 5

4.2. Informative References . . . . . . . . . . . . . . . . .7

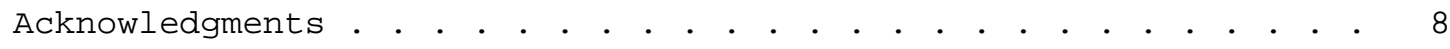

Authors' Addresses . . . . . . . . . . . . . . . . . . . . . 8

1. Introduction

TCP has a long history. Over time, many RFCs have accumulated that describe aspects of the TCP protocol, implementation, and extensions. Some of these have been superseded, are no longer recommended for use, or have simply never seen widespread use.

Sections 6 and 7.1 of the TCP roadmap document [RFC7414] already reclassified a number of TCP extensions as "Historic" and describes the reasons for doing so, but it did not instruct the RFC Editor to change the status of these RFCs in the RFC database. The purpose of this document is to do just that.

In addition, this document reclassifies all other documents mentioned in the TCP roadmap that currently have an "Unknown" status to either "Historic" or "Informational". 


\section{Status Changes}

The following two sections give a short justification why a specific TCP extension or a TCP-related document is being reclassified as "Historic" or "Informational". In addition, the letter code after an RFC number indicates from which original status a particular RFC is changed to "Historic" or "Informational" (see BCP 9 [RFC2026] for an explanation of these categories):

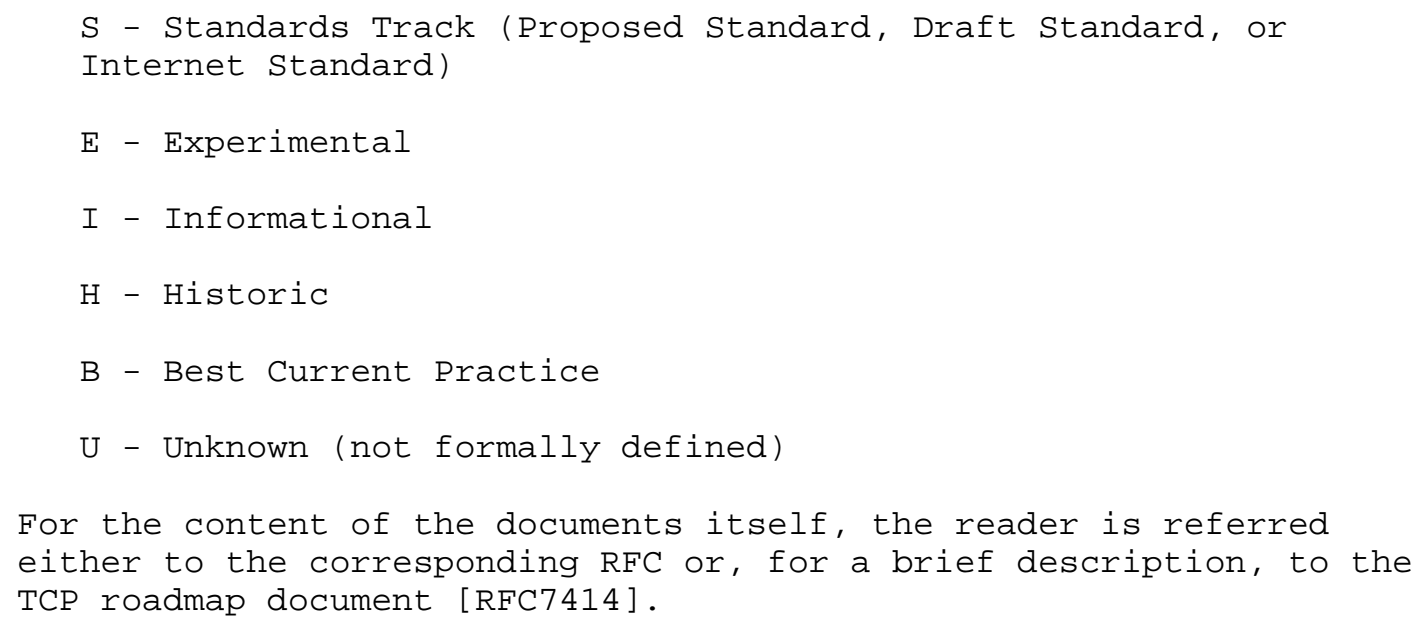


- [RFC879] U, "The TCP Maximum Segment Size and Related Topics" was incorporated into [RFC1122] and [RFC6691]

- [RFC896] U, "Congestion Control in IP/TCP Internetworks" was incorporated into [RFC1122] and [RFC6633]

- [RFC1078] U, "TCP Port Service Multiplexer (TCPMUX)" should be deprecated, because:

* It modifies the TCP connection establishment semantics by also completing the three-way handshake when a service is not available.

* It requires all new connections to be received on a single port, which limits the number of connections between two machines.

* It complicates firewall implementation and management because all services share the same port number.

* There are very limited deployments, and these are not used in an Internet context. (The only reported use is for SGI's Data Migration Facility in private networks.)

- [RFC6013] E, "TCP Cookie Transactions (TCPCT)" should be deprecated (although only published in 2011) because:

* It uses the experimental TCP option codepoints, which prohibit a large-scale deployment.

* [RFC7413] and [TCP-EDO] are alternatives that have more "rough consensus and running code" behind them.

* There are no known wide-scale deployments.

2.2. Moving to "Informational" Status

This document changes the status of the following RFCs to

"Informational" [RFC2026]:

- [RFC700] U, "A Protocol Experiment", which presents a field report about the deployment of a very early version of TCP

- [RFC794] U, "Pre-emption", which recommends that operating systems need to manage their limited resources, which may include TCP connection state

- [RFC814] U, "Name, Addresses, Ports, and Routes", which gives guidance on designing tables and algorithms to keep track of various identifiers within a TCP/IP implementation

- [RFC817] U, "Modularity and Efficiency in Protocol Implementation", which contains general implementation suggestions 
- [RFC872] U, "TCP-on-a-LAN", which concludes that the fear of using TCP on a local network is unfounded

- [RFC889] U, "Internet Delay Experiments", which describes experiments with the TCP retransmission timeout calculation

- [RFC964] U, "Some Problems with the Specification of the Military Standard Transmission Control Protocol", which points out several specification bugs in the US Military's MIL-STD-1778 document, which was intended as a successor to [RFC793]

o [RFC1071] U, "Computing the Internet Checksum", which lists a number of implementation techniques for efficiently computing the Internet checksum

\section{Security Considerations}

This document introduces no new security considerations. Each RFC listed in this document attempts to address the security considerations of the specification it contains.

\section{References}

\subsection{Normative References}

[RFC675] Cerf, V., Dalal, Y., and C. Sunshine, "Specification of Internet Transmission Control Program", RFC 675, DOI 10.17487/RFC0675, December 1974, <http://www.rfc-editor.org/info/rfc675>.

[RFC700] Mader, E., Plummer, W., and R. Tomlinson, "Protocol experiment", RFC 700, DOI 10.17487/RFC0700, August 1974, <http://www.rfc-editor.org/info/rfc700>.

[RFC721] Garlick, L., "Out-of-Band Control Signals in a Host-toHost Protocol", RFC 721, DOI 10.17487/RFC0721, September 1976, <http://www.rfc-editor.org/info/rfc721>.

[RFC761] Postel, J., "DoD standard Transmission Control Protocol", RFC 761, DOI 10.17487/RFC0761, January 1980, <http://www.rfc-editor.org/info/rfc761>.

[RFC794] Cerf, V., "Pre-emption", RFC 794, DOI 10.17487/RFC0794, September 1981, <http://www.rfc-editor.org/info/rfc794>.

[RFC813] Clark, D., "Window and Acknowledgement Strategy in TCP", RFC 813, DOI 10.17487/RFC0813, July 1982, <http://www.rfc-editor.org/info/rfc813>. 
[RFC814] Clark, D., "Name, addresses, ports, and routes", RFC 814, DOI 10.17487/RFC0814, July 1982, <http://www.rfc-editor.org/info/rfc814>.

[RFC816] Clark, D., "Fault isolation and recovery", RFC 816, DOI 10.17487/RFC0816, July 1982, <http://www.rfc-editor.org/info/rfc816>.

[RFC817] Clark, D., "Modularity and efficiency in protocol implementation", RFC 817, DOI 10.17487/RFC0817, July 1982, <http://www.rfc-editor.org/info/rfc817>.

[RFC872] Padlipsky, M., "TCP-on-a-LAN", RFC 872, DOI 10.17487/RFC0872, September 1982, <http://www.rfc-editor.org/info/rfc872>.

[RFC879] Postel, J., "The TCP Maximum Segment Size and Related Topics", RFC 879, DOI 10.17487/RFC0879, November 1983, <http://www.rfc-editor.org/info/rfc879>.

[RFC889] Mills, D., "Internet Delay Experiments", RFC 889, DOI 10.17487/RFC0889, December 1983, <http://www.rfc-editor.org/info/rfc889>.

[RFC896] Nagle, J., "Congestion Control in IP/TCP Internetworks", RFC 896, DOI 10.17487/RFC0896, January 1984, <http://www.rfc-editor.org/info/rfc896>.

[RFC964] Sidhu, D. and T. Blumer, "Some problems with the specification of the Military Standard Transmission Control Protocol", RFC 964, DOI 10.17487/RFC0964, November 1985, <http://www.rfc-editor.org/info/rfc964>.

[RFC1071] Braden, R., Borman, D., and C. Partridge, "Computing the Internet checksum", RFC 1071, DOI 10.17487/RFC1071, September 1988, <http://www.rfc-editor.org/info/rfc1071>.

[RFC1078] Lottor, M., "TCP port service Multiplexer (TCPMUX)", RFC 1078, DOI 10.17487/RFC1078, November 1988, <http://www.rfc-editor.org/info/rfcl078>.

[RFC6013] Simpson, W., "TCP Cookie Transactions (TCPCT)", RFC 6013, DOI 10.17487/RFC6013, January 2011, <http://www.rfc-editor.org/info/rfc6013>. 


\subsection{Informative References}

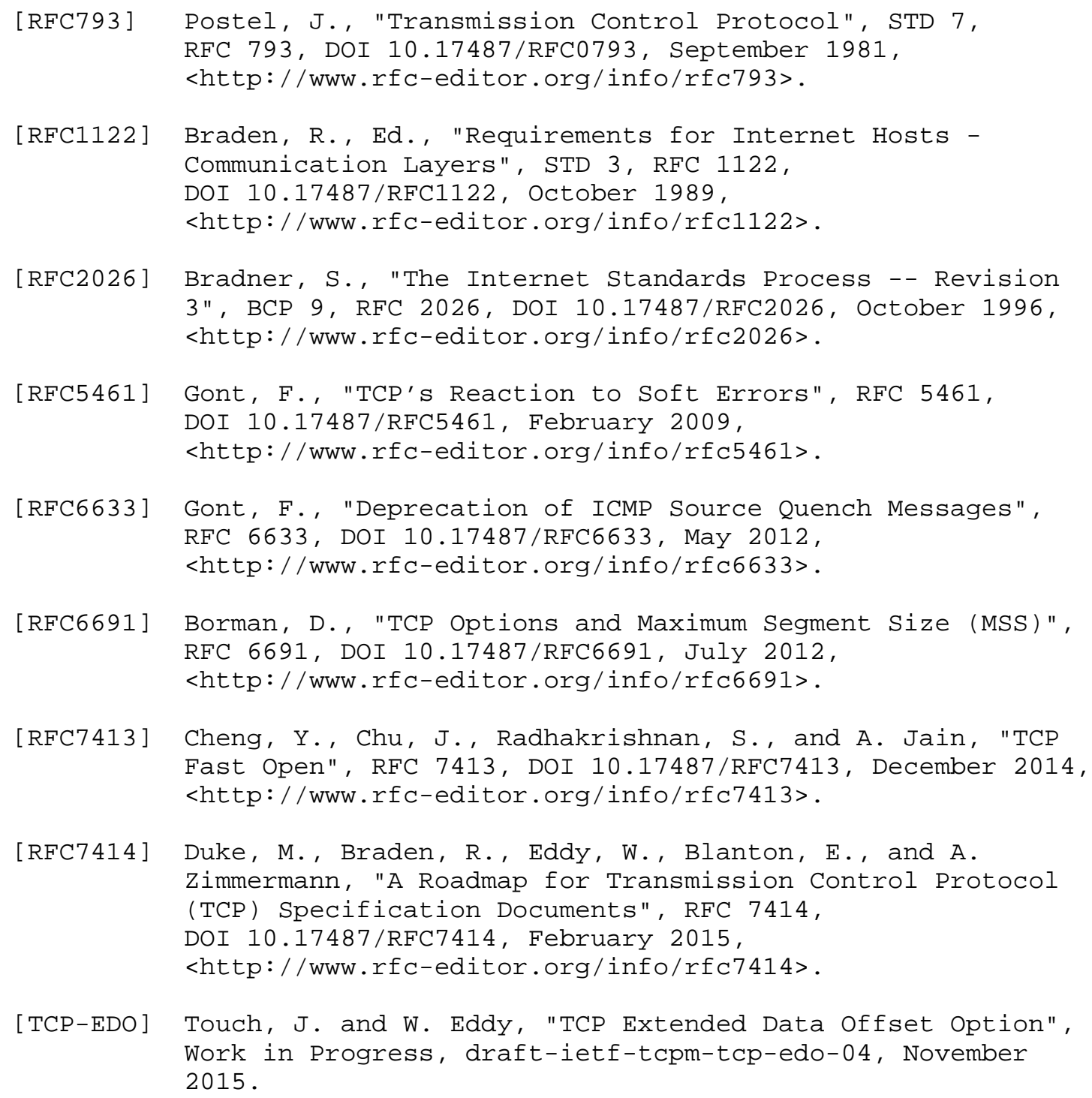




\section{Acknowledgments}

The authors thank John Leslie, Pasi Sarolahti, Richard Scheffenegger, Martin Stiemerling, Joe Touch, Valdis Kletnieks, and Greg Skinner for their contributions.

Lars Eggert has received funding from the European Union's Horizon 2020 research and innovation program 2014-2018 under grant agreement No. 644866 (SSICLOPS). This document reflects only the authors' views, and the European Commission is not responsible for any use that may be made of the information it contains.

Authors' Addresses

Alexander Zimmermann

Email: alexanderdzimmermann.eu.com

Wesley M. Eddy

MTI systems

Suite 170, 18013 Cleveland Parkway

Cleveland, OH 44135

United States

Phone: +1-216-433-6682

Email: wes@mti-systems.com

Lars Eggert

NetApp

Sonnenallee 1

Kirchheim 85551

Germany

Phone: +49151 12055791

Email: lars@netapp.com 